\title{
La familia como factor predictor de la intimidación escolar en Antioquia (Colombia)
}

\author{
Nadia Semenova Moratto Vásqueza ${ }^{\mathrm{a}}$, Dedsy Yajaira Berbesí Fernández ${ }^{\mathrm{b}}$, Natalia Cárdenas \\ Zuluaga $^{c}$, Juan Carlos Restrepo Botero ${ }^{d}$ y Luis Felipe Londoño Ardila
}

\author{
a Magíster en Psicología. \\ Grupo de Investigación en \\ Psicología, Salud y Sociedad. \\ Universidad CES. Medellín, \\ Colombia. \\ b Magíster en Epidemiología. \\ Grupo de Investigación en \\ Epidemiología y Bioestadística. \\ Universidad CES. Medellín, \\ Colombia. \\ c Magíster en Educación y \\ Desarrollo Humano. Grupo de \\ Investigación en Psicología, \\ Salud y Sociedad. Universidad \\ CES. Medellín, Colombia \\ d Doctor en Psicología con \\ orientación en Neurociencia \\ Cognitiva Aplicada. Grupo de \\ Investigación en Psicología \\ Aplicada de la Corporación \\ Universitaria Lasallista \\ e Magister en Educación. Grupo \\ de Investigación en Psicología \\ Aplicada de la Corporación \\ Universitaria Lasallista
}

Correspondencia: Nadia Semenova Moratto. Universidad CES. Medellín, Colombia.

Correo electrónico: nmoratto@ces.edu.co

Recibido el 24 de abril de 2015. Aceptado para su publicación el 11 de junio de 2015.

\begin{abstract}
RESUMEN
Objetivo: Se realizó una investigación que buscaba determinar la prevalencia de la intimidación escolar en Antioquia y la relación de la función familiar en la aparición de este fenómeno.

Diseño del estudio: Estudio transversal comparativo.

Participantes: La población de estudio fueron todos los estudiantes matriculados en los grados de $5^{\circ}$ a $9^{\circ}$ de las instituciones educativas del Departamento de Antioquia durante el año 2013. Para la selección de la muestra final se realizó un muestreo polietápico, para una muestra final total de 3.222 estudiantes.

Mediciones principales: Se evaluó la funcionalidad familiar mediante el instrumento APGAR familiar y la prevalencia de la Intimidación escolar a través del cuestionario CIE A abreviado, conformado por tres categorías de evaluación: victimización por intimidación, sintomatología asociada a la Intimidación escolar como ansiedad, depresión, estrés postraumático y también efectos sobre autoestima y, finalmente, el tercer componente, intimidación por respondientes, el cual explora variables sobre formas de violencia por parte de las personas que realizan acciones de intimidación escolar a otros pares.

Resultados: El $46 \%$ de los escolares con riesgo alto de bullying, el $43 \%$ con riesgo medio y el $25 \%$ con riesgo bajo, tienen disfunción familiar moderada a grave. A su vez, la disfunción familiar grave está fuertemente asociada con el riesgo de intimidación escolar en los escolares de Antioquia, denotando con ello que los escolares expuestos a disfunción familiar tienen menos estrategias para relacionarse, sin utilizar la agresión.

Conclusiones: Las relaciones estrechas entre los miembros de la familia se encuentran fuertemente relacionadas con las estrategias de vinculación, que permiten afrontar situaciones conflictivas en diversos escenarios, entre ellos el de mayor demanda en la vida cotidiana de los adolescentes, como lo es el escolar. Por tanto, con el acompañamiento decidido de la familia, podría pensarse que los niños y adolescentes resuelvan las situaciones de agresión escolar con estrategias diferentes al bullying.
\end{abstract}

PALABRAS CLAVE: Intimidación escolar. Familia. Función familiar. Socialización.

\section{ABSTRACT \\ The family as a predictor of bullying in Antioquia (Colombia)}

Objective: To determine the prevalence of bullying in Antioquia and the relationship of the family role in the development of this phenomenon.

Study design: Cross-sectional study.

Participants: Students enrolled in grades 5 th to 9 th of educational institutions in the Department of Antioquia in 2013. The final sample was made through a multistage sampling with a total of 3222 students.

Main Measures: The family functioning was assessed using the familiar APGAR instrument, and the prevalence of school bullying through the questionnaire CIE A (abbreviated) that consists of three assessment categories: victimization, symptoms associated with school bullying as: anxiety, depression, PTSD and self-esteem, and finally, Intimidation, which explores variables on forms of violence by people performing bullying actions to other pairs .

Results: $46 \%$ of the students with high risk of bullying, $43 \%$ of students with medium risk, and $25 \%$ of students with low risk have moderate to severe family dysfunction. At the same time, severe family dysfunction is strongly associated with the risk of bullying at school, meaning that children exposed to family dysfunction have fewer strategies to interact without using aggression.

Conclusions: The close relationships between family members are closely related to linking strategies that allow addressing conflicts in different contexts, including the school as the most important in the daily lives of adolescents. Therefore, with the strong support of the family, children and adolescents resolve situations of aggression with strategies different than bullying. KEY WORDS: School Bullying. Family. Family functioning. Socialization 


\section{INTRODUCCIÓN}

La intimidación o acoso escolar es una realidad que ha existido en los colegios o escuelas desde siempre y se ha considerado un proceso normal dentro de una "cultura del silencio" que ayuda a su permanencia1. Pero en los últimas décadas esta intimidación se ha descrito como un problema generalizado de salud pública, aunque en la literatura actual sobre la intimidación no existe un consenso ni una definición operativa sobre el concepto y esta puede variar según el marco teórico ${ }^{2}$.

A este respecto, Ramírez ${ }^{3}$ define el "bullying" (el cual puede ser equiparado con el acoso escolar) como "una forma de maltrato, normalmente intencionado y perjudicial de un estudiante hacia otro compañero, generalmente más débil, al que convierte en su víctima habitual; suele ser persistente, y puede durar semanas, meses e incluso años". Puede verse cómo existe una aproximación de símil entre "bullying" y acoso escolar, mientras que otros autores logran diferenciarlos.

Para el caso que compete en el presente artículo, se entenderá el reconocido fenómeno de "bullying" como intimidación escolar, que para Magendzo 4 es "el hostigamiento, el acoso y/o la amenaza sistemática de parte de un estudiante o de un grupo de estudiantes hacia un alumno o alumna". La intimidación ha sido identificada como uno de los tantos comportamientos violentos que existen en el ámbito escolar. Al igual que otros actos violentos, ha sido vinculada con situaciones de conflicto y con la agresividad. Su fin es lesionar a otro, producir daño, destruir, contrariar o humillar ${ }^{4}$.

Si bien puede pensarse que es en la escuela donde este fenómeno tiene su aparición y por tanto este espacio, considerado por tradición como aquel llamado a promover la relación social de los sujetos que allí se encuentran, ya no se torna seguro para el encuentro con el otro, es menester retornar al lugar natural de socialización primaria, es decir a la familia.

La familia constituye el eje fundamental en diversas áreas del sujeto, tales como la socialización, alimentación o protección, entre otros. Por lo tanto, se le ve como espacio fundante en la construcción de las pautas de relación del individuo, y en las apreciaciones iniciales que este tenga del mundo. Es así, como Gómez et al. ${ }^{5}$ anotan que los modelos de relación aprendidos en la familia, tanto por experiencia directa como vicaria, las escalas de valores y los estilos educativos de los padres llevan a los hijos a la construcción de sus propias escalas de valores y estilos de relación; y, como las personas hacemos una selección perceptiva para defendernos de la disonancia cognitiva y no sentirnos mal, tendemos a elegir de forma más o menos automática aquellos elementos del medio que tienden a confirmar que nuestra perspectiva es válida.

Como puede denotarse, los miembros de la fami- lia a través de los roles establecidos por cada uno de ellos y del despliegue de los mismos a través de las relaciones en la intimidad familiar, propician que los niños asuman que dichos comportamientos son los adecuados e importantes para ser imitados por ellos, en aras de enfrentar la propia vida en cualquier escenario. En este sentido, Cerezo sostiene que la familia influye de gran manera en el desarrollo de comportamientos violentos, siendo los agresores inicialmente sometidos a experiencias de maltrato, sufrido u observado ${ }^{3}$.

Así mismo, se encuentra que, entre los principales antecedentes familiares que precipitan conductas agresivas y lesivas en los adolescentes, están la ausencia de una relación afectiva, cálida y segura por parte de los padres (especialmente por la madre), actitudes negativas o escasa disponibilidad para atender al niño y fuertes dificultades para enseñar límites, combinando la permisividad ante conductas antisociales con el frecuente empleo de medidas coercitivas autoritarias, utilizando casi siempre el castigo físico ${ }^{6}$.

La escuela y la familia, por tanto, como espacios de socialización de los niños y adolescentes, son fundamentalmente los encargados de promover en éstos diversas habilidades que impliquen expresión de afecto, enunciación adecuada y divergente de pensamientos, así como estrategias de resolución de conflictos diferentes a la agresión entre pares y abuso del poder.

En este contexto se realizó esta investigación, cuyo objetivo fundamental fue determinar la prevalencia de la intimidación escolar en Antioquia y la relación de la función familiar en la aparición de este fenómeno.

\section{METODOLOGÍA}

Se realizó un estudio de tipo transversal en las instituciones educativas de las nueve subregiones de Antioquia. La población del estudio estuvo constituida por el total de estudiantes matriculados en los grados $5^{\circ}$ a $9^{\circ}$, un total estimado para 2013 de 610.000. Se realizó un muestreo polietápico, en la primera etapa se seleccionaron los municipios, a través de un muestreo sistemático; en la segunda etapa se seleccionaron las instituciones educativas, de acuerdo a una probabilidad proporcional; y en la tercera etapa se seleccionaron los grupos, utilizando un muestreo aleatorio simple.

El tamaño de muestra fue calculado con base en la fórmula para la estimación de una proporción poblacional, para lo cual se utilizó un nivel de confianza del $95 \%$, una precisión del $5 \%$ y una prevalencia esperada de acoso escolar del 10,0\%7.

Los criterios de inclusión de la muestra fueron el ser estudiantes matriculados en los colegios seleccionados y pertenecer a los grados escolares de 
$5^{\circ}$ a $9^{\circ}$. El personal de trabajo de campo fue seleccionado con base en su capacitación y experiencia para las tareas de recolección de la información y manejo de los instrumentos a aplicar.

El primer instrumento utilizado para valorar el funcionamiento familiar fue el APGAR, sigla derivada de los cinco componentes (en inglés) de la función familiar: adaptabilidad (adaptability), que se define como la utilización de los recursos intra y extrafamiliares para resolver los problemas cuando el equilibrio de la familia se ve amenazado por un factor de estrés durante un período de crisis; cooperación (partnertship), como la participación en la toma de decisiones y responsabilidades, lo cual define el grado de poder de los miembros de la familia; desarrollo (growth), como la posibilidad de maduración emocional y física, así como de autorrealización de los miembros de la familia, por el apoyo mutuo; afectividad (affection), como la relación de amor y atención entre los miembros de la familia, y capacidad resolutiva (resolve), como el compromiso o determinación de dedicar tiempo (espacio, dinero) a los otros miembros de la familia.

Al tiempo, se aplicó el cuestionario de intimidación escolar CIE A abreviado, de Berbesí ${ }^{8}$, constituido por tres categorías de evaluación: la primera, denominada victimización por intimidación; la segunda, denominada sintomatología, la cual agrupa variables sobre síntomas de ansiedad, depresión, estrés postraumático y también efectos sobre autoestima; y, finalmente, el tercer componente, la intimidación por respondientes, que recoge variables sobre formas de violencia por parte de las personas que realizan acciones de intimidación escolar a otros pares.

Se realizó un análisis multivariado calculando un modelo de regresión logística multinomial (explicativo del riesgo de "bullying") calculando los OR crudos y después los OR ajustados con el método de Main effect, con sus intervalos de confianza del $95 \%$ y pruebas de significación estadística menor del $5 \%$. La categoría de referencia fue tener funcionabilidad familiar, las categorías que se compararon fueron disfunción grave, moderada y baja.

\section{RESULTADOS \\ Características sociodemográficas (tabla 1)}

La muestra total encuestada fueron 3.222 estudiantes en Antioquia, con edades comprendidas entre los 9 y los 18 años. El 58,3 \% se encuentra entre los 13 y 16 años, seguido de un 36,6\% correspondiente a las edades entre 9 y 12 años. La distribución por sexo fue similar con un 48,5\% de niños y un $51,5 \%$ de niñas. En relación con el grado escolar, se encuentra que el $19 \%$ estaba cursando quinto, sexto y noveno y el $20 \%$ séptimo y octavo grado.

\begin{tabular}{lcc}
\hline & Frecuencia & $\%$ \\
\hline Edad & 1180 & $36,6 \%$ \\
9 a 12 & 1878 & $58,3 \%$ \\
$13-16$ & 164 & $5,1 \%$ \\
$>17$ & 3222 & $100,0 \%$ \\
Total & & \\
Sexo & 1562 & $48,5 \%$ \\
Niños & 1660 & $51,5 \%$ \\
Niñas & 3222 & $100 \%$ \\
Total & & \\
Escolaridad & 612 & $19,4 \%$ \\
$5^{\circ}$ & 623 & $19,7 \%$ \\
$6^{\circ}$ & 658 & $20,8 \%$ \\
$7^{\circ}$ & 645 & $20,4 \%$ \\
$8^{\circ}$ & 619 & $19,6 \%$ \\
$9^{\circ}$ & 3157 & $100 \%$ \\
\hline Total & &
\end{tabular}

Tabla 1. Características sociodemográficas de los escolares en Antioquia 


\section{Prevalencia de la intimidación escolar (tabla 2)}

El $64,1 \%$ de los escolares tienen bajo riesgo de intimidación escolar y un $28,3 \%$ riesgo medio. Con una menor proporción se encuentra un 4,0\% de niños y adolescentes con riesgo alto de Intimidación Escolar. El 92,4 \% de los escolares son clasificados con riesgo bajo y medio de Intimidación Escolar.

\section{Relación entre la disfunción familiar y la intimi-} dación escolar (tabla 3)

Un 46,2 \% de los escolares con disfunción moderada a grave presenta riesgo alto de Intimidación
Escolar. Seguido de un 45,9 \% de niños y adolescentes con riesgo medio de Intimidación.

Como se aprecia en la tabla IV, los escolares con disfunción familiar grave tienen seis veces la probabilidad de presentar riesgo alto de Intimidación Escolar (OR: 5,98; IC 2,47-14,4); cuatro veces la probabilidad de presentar riesgo medio y dos veces riesgo bajo de intimidación escolar, observando un efecto dosis-respuesta, donde a mayor exposición a problemas y dificultades familiares, mayor riesgo de no poseer estrategias para afrontar dificultades en el ámbito escolar. Estas diferencias fueron estadísticamente significativas.

\begin{tabular}{|c|c|c|c|c|c|c|c|c|}
\hline \multirow{2}{*}{$\begin{array}{l}\text { Intimidación } \\
\text { escolar }\end{array}$} & \multicolumn{3}{|c|}{ Hombres } & \multicolumn{3}{|c|}{ Mujeres } & \multicolumn{2}{|c|}{ Total } \\
\hline & $\mathbf{n}$ & $\%$ & IC 95\% & $\mathbf{n}$ & $\%$ & IC 95\% & $\mathbf{n}$ & $\%$ \\
\hline Sin riesgo & 43 & 3,1 & $(2,1-4,1)$ & 58 & 4,0 & $(2,9-4,9)$ & 101 & 3,6 \\
\hline Riesgo bajo & 856 & 62,6 & $(59,9-65,1)$ & 960 & 65,6 & $(63,1-68-0)$ & 1816 & 64,1 \\
\hline Riesgo medio & 410 & 30,0 & $(27,5-32,4)$ & 392 & 26,8 & $(24,4-29,0)$ & 802 & 28,3 \\
\hline \multirow[t]{2}{*}{ Riesgo alto } & 59 & 4,3 & $(3,2-5,4)$ & 53 & 3,6 & $(2,6-4,6)$ & 112 & 4,0 \\
\hline & 1368 & 100 & & 1463 & 100 & & 2831 & 100 \\
\hline
\end{tabular}

Tabla 2. Prevalencia de la Intimidación Escolar por sexos

\begin{tabular}{|c|c|c|c|c|c|c|c|c|c|c|c|c|c|}
\hline \multirow{3}{*}{$\begin{array}{l}\text { APGAR } \\
\text { Familiar }\end{array}$} & \multicolumn{13}{|c|}{ Intimidación Escolar } \\
\hline & \multicolumn{3}{|c|}{ Sin riesgo } & \multicolumn{3}{|c|}{ Riesgo bajo } & \multicolumn{3}{|c|}{ Riesgo medio } & \multicolumn{3}{|c|}{ Riesgo alto } & \multirow{2}{*}{$\begin{array}{c}\text { Total } \\
\mathrm{n}\end{array}$} \\
\hline & $\mathrm{n}$ & $\%$ & IC 95\% & $\mathrm{n}$ & $\%$ & IC 95\% & $\mathrm{n}$ & $\%$ & IC 95\% & $\mathrm{n}$ & $\%$ & IC 95\% & \\
\hline $\begin{array}{c}\text { Disfunción } \\
\text { grave }\end{array}$ & 10 & $2,3 \%$ & $(0,7-3,8)$ & 242 & $55,8 \%$ & $(50,9-60,5)$ & 154 & $35,5 \%$ & $(30,8-40,1)$ & 28 & $6,5 \%$ & $4,0-8,8)$ & 434 \\
\hline $\begin{array}{l}\text { Disfunción } \\
\text { moderada }\end{array}$ & 11 & $3,1 \%$ & $(1,1-5,0)$ & 201 & $56,9 \%$ & $(51,6-62,2)$ & 126 & $35,7 \%$ & $(30,5-40,8)$ & 15 & $4,2 \%$ & $2,0-6,4)$ & 353 \\
\hline $\begin{array}{l}\text { Disfunción } \\
\text { leve }\end{array}$ & 15 & $2,4 \%$ & $(1,1-3,7)$ & 406 & $65,8 \%$ & $(23,6-30,8)$ & 168 & $27,2 \%$ & $(23,6-30,8)$ & 28 & $4,5 \%$ & $(2,8-6,2)$ & 617 \\
\hline Funcional & 47 & $5,4 \%$ & $(3,8-6,9)$ & 609 & $69,9 \%$ & $(66,8-73,0)$ & 193 & $22,2 \%$ & $(19,3-24,9)$ & 22 & $2,5 \%$ & $(1,4-3,6)$ & 871 \\
\hline
\end{tabular}

Tabla 3. Funcionalidad familiar e intimidación escolar en escolares de Antioquia

\begin{tabular}{lcccccc}
\hline Intimidación Escolar & \multicolumn{2}{c}{ Riesgo bajo } & \multicolumn{2}{c}{ Riesgo medio } & \multicolumn{2}{c}{ Riesgo alto } \\
\cline { 2 - 7 } Apgar familiar & OR & IC (OR) & OR & IC (OR) & OR & IC (OR) \\
\hline Disfunción grave & 1,86 & $0,92-3,75$ & 3,75 & $1,83-7,66$ & 5,98 & $2,47-14,4$ \\
Disfunción moderada & 1,41 & $0,71-2,77$ & 2,78 & $1,39-5,58$ & 2,91 & $1,15-7,37$ \\
Disfunción bajo & 2,08 & $1,15-3,78$ & 2,72 & $1,47-5,05$ & 3,98 & $1,78-8,9$ \\
Sin riesgo & 1,00 & - & 1,00 & - & 1,00 & - \\
\hline
\end{tabular}

Tabla 4. Funcionalidad familiar y riesgo de intimidación escolar en escolares de Antioquia 


\section{DISCUSIÓN}

Conceptualizar la familia y las funciones que esta cumple al interior de la sociedad ha sido una tarea de las últimas décadas, y cada vez más las concepciones de la misma se centran en la función que tiene como institución que moldea a cada uno de sus miembros. Para que esto cobre sentido es necesario ver a la familia como una institución que, a lo largo de los años, no se mantiene estática, sino que persiste continuamente en un proceso de transformación, acorde al contexto y a los sujetos que en ella se sostienen. La familia, por tanto, vista desde Lasch $^{9}$, se ha convertido en el refugio de la sociedad -que es cada vez más fría-, en el lugar de reposo después del trabajo, donde se esperan encontrar los afectos que ya no se encuentran en lo público. A la vez, se convierte en el lugar donde se puede ser "uno mismo" sin la represión que lo público ejerce sobre los comportamientos y, sobre todo, en los sentimientos. La afectividad familiar contribuye a mantener el equilibrio emocional de los miembros y a la adaptación funcional de estos en la sociedad, por lo que no puede quedar marginado del análisis social.

Pensar en la familia como espacio de socialización primario adquiere relevancia e implica que las enseñanzas allí construidas modularán las formas y estrategias de relación posterior del sujeto con el contexto. Es por eso que pensar en la familia y su funcionalidad en relación con cómo los niños y adolescentes pueden o no construir relaciones en el medio escolar, cobra relevancia. Al respecto, Havighurst ${ }^{10}$ sostiene que "cuando el niño ingresa en la escuela no es ya una personalidad neutral, sin formación previa, que pueda ser modelada en muchas de las diversas formas posibles. No es ya, como lo fue en el momento de nacer, una criatura no socializada y sin experiencia, sin actitudes ni objetivos ni ideas propias. Cuando penetra a la sala de clases es, por el contrario, un producto de la educación familiar y tiene tras de sí una larga historia social. Aun cuando ejerza una fuerte influencia y cambie su comportamiento en muchos e importantes aspectos, la escuela nunca opera sola, lo hace siempre en relación con la familia".

Considerar la disfunción familiar como factor de fuerte asociación con el riesgo de intimidación escolar en los jóvenes cobra sentido, puesto que si se piensa que la familia constituye la primera instancia de socialización de los niños, implica el que allí estos adquieren los repertorios básicos que posteriormente reproducirán en otros escenarios, como en la escuela. En ella, no solo tendrá que enfrentar adultos con concepciones diversas, sino a los grupos de pares que exigirán de él intercambios emocionales y de acción. En este sentido, es posible decir que las acciones violentas realizadas en la escuela responden a prácticas cotidianas de agresiones diversas dentro de la unidad familiar, donde existen "estilos educativos autoritarios, con poco afecto, donde las normas se imponen y solo responden al interés del adulto" ${ }^{11}$.

Así, retomar el concepto de roles es significativo, dado que estos son asumidos por cada uno de los miembros que conforman a la familia, puesto que se constituyen en el comportamiento que se espera de cada uno de ellos. Este término está relacionado con el status, puesto que "un status es una serie de privilegios y obligaciones; un rol es el desempeño de esta serie de privilegios" ${ }^{12}$. Los roles, de alguna manera le permiten comprender a las personas cómo debe ser su comportamiento y su postura de acuerdo con la situación en la que se encuentre.

Encontrar, por tanto, que el $46 \%$ de los escolares con riesgo alto de "bullying", el $43 \%$ con riesgo medio y el $25 \%$ con riesgo bajo, tienen disfunción familiar moderada a grave, implica que a mayor disfunción familiar, mayor riesgo de "bullying". Es decir, se observa un efecto dosis-respuesta, donde a mayor exposición a problemas y dificultades familiares, mayor riesgo de no poseer estrategias para afrontar dificultades en el ámbito escolar. Según Villarroel y Sánchez ${ }^{13}$, dos factores determinantes en la socialización del niño son la familia y la escuela, siendo la familia el primer mundo social que encuentra el niño y la niña; a través de este agente se los introduce en las relaciones íntimas y personales, proporcionándoles sus primeras experiencias, como la de ser tratados como individuos distintos. Igualmente, se convierte en el primer grupo referencial de normas y valores que el niño adopta como propias y que en el futuro le ayudarán a emitir juicios sobre sí mismo. Todas estas experiencias sociales que los niños y niñas vivencian dentro del núcleo familiar son la base para la formación de su personalidad. La familia es la responsable del proceso de transmisión cultural inicial y su papel principal es introducir a sus miembros en las diversas normas, pautas y valores que en un futuro le permitirán vivir en sociedad, porque es allí donde se aprenden por primera vez los tipos de conductas y actitudes consideradas socialmente aceptables y apropiadas según género.

Como factores que se han asociado a la aparición del "bullying", están las características de las familias de los agresores o agresoras. En general, los padres y madres exhiben pocas destrezas para solucionar problemas, así como para mantener relaciones con otros adultos; son inconsistentes y/o abusivos con la disciplina, y no están interesados en conocer siempre el paradero de sus hijos o hijas $^{14-17}$. Si bien esta caracterización no fue indagada en el proceso de investigación, sí da pautas esenciales para rastrear aspectos coadyuvantes en la función o disfunción de la familia.

Como puede verse, los comportamientos de los padres y demás personas significativas para los niños serán los potenciales modelos evocados en el proceso de socialización de los adolescentes. Por tanto, cuanto mayores sean las estrategias de so- 
cialización asertivas y de resolución adecuada de problemas, mejores niveles de relación serán sostenidas y mantenidas en el escenario escolar, ante esto, algunos autores sostienen que "la agresividad de los padres apoya el aumento de agresividad de los hijos" 18 .

Se reconoce, por tanto, que los niños y adolescentes requieren de un ambiente propicio para su desarrollo intelectual, físico y social, donde los roles de los padres sean modelos primarios de imitación, caracterizados por amplias habilidades para enfrentar situaciones problema en la vida cotidiana, expresión de afectos y retroalimentación constante a los hijos.

Esta investigación, contó con limitaciones que le generan restricción a los resultados expuestos pero no invalidan sus resultados, tales como que los datos provienen de una encuesta autodiligenciada, anónima y confidencial, pudiendo tener imprecisiones basadas en la honestidad de la respuesta y quedando preguntas sin responder por parte de los participantes; no es posible realizar asociaciones de causa efecto entre la intimidación escolar y los factores encontrados debido al tipo de diseño, en los estudios transversales los datos del evento y la exposición son medidos en el mismo momento.

Se puede concluir que el riesgo alto y moderado de intimidación escolar se encuentra fuertemente asociado con la disfunción familiar. Por tanto, las pocas estrategias modeladas de vinculación con el otro y con la resolución de problemas se encuentran influenciadas por la agresión y ello se ve replicado por los niños y adolescentes en el contexto escolar.

\section{BIBLIOGRAFÍA}

1. Trautmann MA. Maltrato entre pares o "bullying": Una visión actual. Rev Chil Pediatr. 2008;79(1):13-20.

2. Liu J, Graves N. Childhood bullying: A review of constructs, concepts, and nursing implications. Public Health Nursing. 2011;28(6):556-68.

3. Ramírez FC. Análisis comparativo de variables socio-afectivas diferenciales entre los implicados en bullying. Estudio de un caso de víctima-provocador. Anu Psicol Clin Salud. 2006;2:27-34.
4. Magendzo A. La intimidación entre estudiantes: Una realidad presente en los establecimientos educacionales [Internet]. Disponible en: http://www.educarchile.cl/humano2/ h2/asp/Magendzo.pdf [consultado el 25 de abril de 2015].

5. Gómez A, Gala F, Lupiani M, Bernalte A, Miret M, Lupiani $\mathrm{S}$, et al. El "bullying" y otras formas de violencia adolescente. Cuad Med Forense. 2005;13(48-49):165-177.

6. Díaz-Aguado Jalon MJ. La violencia entre iguales en la adolescencia y su prevención desde la escuela. Psicothema. 2005;17(4):549-58.

7. Chaux E, Bustamante A, Castellanos M, Chaparro MP, Jiménez M. Intimidación escolar y el rol de los testigos. Educación en valores y ciudadanía desde una perspectiva cotidiana. Bogotá: Instituto para el desarrollo y la innovacion educativa IDIE; 2009. p.83-98.

8. Berbesí Fernández D. Validación de un cuestionario breve para detectar intimidación escolar. CES Psicol. 2012;5(2):70-8.

9. Lasch C. Refugio en un mundo despiadado: reflexión sobre la familia contemporánea [Internet]. Gedisa; 1996. Disponible en: http://dialnet.unirioja.es/servlet/ libro?codigo=97091 [consultado el 25 de abril de 2015]

10. Havighurst R. La sociedad y la educación en América Latina. Buenos Aires: Editorial Universitaria de Buenos Aires; 1962.

11. Serrano A. Acoso y violencia en la escuela: cómo detectar, prevenir y resolver el bullying. Barcelona: Ariel; 2006.

12. Viveros Chavarría E. Dinámicas internas de las familias con jefatura femenina y menores de edad en conflicto con la Ley Penal: características interaccionales. Facultad de Desarrollo Familiar. Línea de Investigación Calidad de Vida. Medellín: Fundación Universitaria Luis Amigó; 2006.

13. Villarroel Rosende G, Sánchez Segura X. Relación Familia y Escuela: Un estudio comparativo en la ruralidad. Estudios Pedagógicos, núm. 28, 2002, pp.123-141. Universidad Austral de Chile Valdivia, Chile. Disponible en: http://www. redalyc.org/articulo.oa?id=173513847007 [consultado el 25 de abril de 2015]

14. Olweus D. Conductas de acoso y amenaza entre escolares. Madrid: Morata; 1998.

15. Olweus D. Olweus' core program against bullying and antisocial behavior: a teacher handbook. Bergen Norway: The author; 2001.

16. Cabezas Pizarro H, Monge Jiménez I. Maltrato entre iguales en la escuela costarricense. Rev Educ. 2007;31(1):13544.

17. Paredes MT, Álvarez MC, Lega LI, Vernon A. Estudio exploratorio sobre el fenómeno del "Bullying" en la ciudad de Cali, Colombia. Revista Latinoamericana de Ciencias Sociales, Niñez y Juventud. 2008;6(1):295-317.

18. Cummings EM, Goeke-Morey MC, Papp LM, Dukewich TL. Children's responses to mothers' and fathers' emotionality and tactics in marital conflict in the home. J Fam Psychol. 2002;16(4):478-92. 